\title{
Geometrically distinct solutions of nonlinear elliptic systems with periodic potentials
}

\author{
ZhiPENG YANG(D AND YUANYANG YU
}

\begin{abstract}
In this paper, we study the following nonlinear elliptic systems:

$$
\begin{cases}-\Delta u_{1}+V_{1}(x) u_{1}=\partial_{u_{1}} F(x, u) & x \in \mathbb{R}^{N}, \\ -\Delta u_{2}+V_{2}(x) u_{2}=\partial_{u_{2}} F(x, u) & x \in \mathbb{R}^{N},\end{cases}
$$

where $u=\left(u_{1}, u_{2}\right): \mathbb{R}^{N} \rightarrow \mathbb{R}^{2}, F$ and $V_{i}$ are periodic in $x_{1}, \ldots, x_{N}$ and $0 \notin \sigma\left(-\Delta+V_{i}\right)$ for $i=1,2$, where $\sigma\left(-\Delta+V_{i}\right)$ stands for the spectrum of the Schrödinger operator $-\Delta+V_{i}$. Under some suitable assumptions on $F$ and $V_{i}$, we obtain the existence of infinitely many geometrically distinct solutions. The result presented in this paper generalizes the result in Szulkin and Weth (J Funct Anal 257(12):3802-3822, 2009).
\end{abstract}

Mathematics Subject Classification. 35J47, 35J50.

Keywords. Nonlinear elliptic systems, Geometrically distinct solutions, Variational methods.

1. Introduction and main results. In this paper, we study the multiplicity of solutions for the nonlinear elliptic systems:

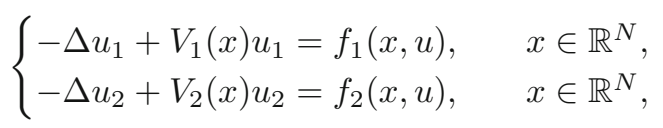

where $f:=\left(f_{1}, f_{2}\right)=\partial_{u} F$ and $F: \mathbb{R}^{N} \times \mathbb{R}^{2} \rightarrow \mathbb{R}$. This type of systems arises when one considers standing wave solutions of time-dependent 2-coupled Schrödinger systems of the form

$$
\left\{\begin{array}{l}
i \frac{\partial \phi_{1}}{\partial t}=-\Delta \phi_{1}+a_{1}(x) \phi_{1}-g_{1}(x,|\phi|) \phi_{1}, \\
i \frac{\partial \phi_{2}}{\partial t}=-\Delta \phi_{2}+a_{2}(x) \phi_{2}-g_{2}(x,|\phi|) \phi_{2},
\end{array}\right.
$$

where $\phi=\left(\phi_{1}, \phi_{2}\right), i$ is the imaginary unit, $a_{i}(x)$ is a potential function, $g_{i}$ is a coupled nonlinear function modeling various types of the interaction effect 
among many particles. System (1.2) has applications in many physical problems, especially in nonlinear optics and in Bose-Einstein condensates theory for multispecies Bose-Einstein condensates (see $[1,10,14]$ ). A standing wave solution of system (1.2) is a solution of the form

$$
\phi_{i}(x, t)=e^{-i \lambda_{i} t} u_{i}(x), \lambda_{i} \in \mathbb{R}, t>0,
$$

and $\left(u_{1}, u_{2}\right)$ solves the system (1.1) with $V_{i}(x)=a_{i}(x)-\lambda_{i}, f_{i}(x, u)=g_{i}(x,|u|) u_{i}$ for $i=1,2$.

System (1.2) has been studied by some authors quite recently. In a bounded smooth domain $\Omega \subset \mathbb{R}^{N}$, the similar systems were extensively studied by some authors, see for instance $[3,5-7,19]$ and the references therein. The problem settled on the whole space $\mathbb{R}^{N}$ was also considered recently in some works. One of the main difficulties of this problem is the lack of the compactness of the Sobolev embedding. And the second difficulty is that the negative definite space of the quadratic form which appears in the energy functional is infinitely dimensional, i.e., the energy functional is strongly indefinite. There are many different conditions and methods involved to avoid these difficulties, we refer to $[4,8,9,16,24,25]$ and the references therein.

Recall that the spectrum $\sigma(-\Delta+V)$ of $-\Delta+V$ is purely continuous and may contain gaps, i.e., open intervals free of spectrum (see [18]). In [21], Szulkin and Weth considered the following Schrödinger equation:

$$
-\Delta u+V(x) u=f(x, u) x \in \mathbb{R}^{N},
$$

and proved that Eq. (1.3) possesses a ground state solution under the assumption $0 \notin \sigma(-\Delta+V)$. Later, Mederski [12] considered the system of coupled Schrödinger equations as follows:

$$
-\Delta u_{i}+V_{i}(x) u_{i}=\partial_{u_{i}} F(x, u), \quad x \in \mathbb{R}^{N}, i=1,2, \ldots, K,
$$

where $F$ and $V_{i}$ are periodic in $x, 0 \notin \sigma\left(-\Delta+V_{i}\right), i=1,2, \ldots, K$, and proved the existence of a ground state solution based on a new linking-type result involving the Nehari-Pankov manifold.

Inspired by the above facts, more precisely by $[12,21]$, the aim of this paper is to study the existence and multiplicity of nontrivial solutions to system (1.1) via variational methods. As far as we know, it seems that this problem was not considered in literature before.

We assume that $V$ and $f$ satisfy the following hypotheses:

$(V) V_{i} \in C\left(\mathbb{R}^{N}, \mathbb{R}\right), 1$-periodic in $x_{1}, \ldots, x_{N}$, and $0 \notin \sigma\left(-\Delta+V_{i}\right)$ for $i=1,2$;

$\left(f_{1}\right) f_{i}: \mathbb{R}^{N} \times \mathbb{R}^{2} \rightarrow \mathbb{R}$ is continuous with respect to the second variable, 1 -periodic in $x_{1}, \ldots, x_{N}$;

$\left(f_{2}\right)$ there are $2<p<2^{*}=\frac{2 N}{(N-2)_{+}}$and $c_{0}>0$ such that $|f(x, u)| \leq c_{0}(1+$ $\left.|u|^{p-1}\right)$ for all $(x, u) \in \mathbb{R}^{N} \times \mathbb{R}^{2}$;

$\left(f_{3}\right) \lim _{|u| \rightarrow 0} \frac{|f(x, u)|}{|u|}=0$ and $\lim _{|u| \rightarrow \infty} \frac{F(x, u)}{|u|^{2}}=\infty$ uniformly in $x \in \mathbb{R}^{N}$;

$\left(f_{4}\right) \frac{1}{2} f(x, u) u \geq F(x, u)>0$ for all $(x, u) \in \mathbb{R}^{N} \times \mathbb{R}^{2} \backslash\{0\}$;

$\left(f_{5}\right)$ if $f(x, u) v=f(x, v) u>0$, then $F(x, u)-F(x, v) \leq \frac{(f(x, u) u)^{2}-(f(x, u) v)^{2}}{2 f(x, u) u}$.

If in addition, $F(x, u) \neq F(x, v)$, then the strict inequality holds. 
We note that if $u_{0}$ is a solution of (1.1), then so are all elements of the orbit of $u_{0}$ under the action of $\mathbb{Z}^{N}, \mathcal{O}(u):=\left\{u(\cdot-k): k \in \mathbb{Z}^{N}\right\}$. Two solutions $u_{1}$ and $u_{2}$ are said to be geometrically distinct if $\mathcal{O}\left(u_{1}\right)$ and $\mathcal{O}\left(u_{2}\right)$ are disjoint.

We now describe our main result.

Theorem 1.1. Assume that $(V)$ and $\left(f_{1}\right)-\left(f_{5}\right)$ hold, then the following conclusions are true:

(i) system (1.1) has a ground state solution;

(ii) if in addition $F$ is even in $u$, that is, $F(x, u)=F(x,-u)$ for any $(x, u) \in$ $\mathbb{R}^{N} \times \mathbb{R}^{2}$, then system (1.1) admits infinitely many pairs of geometrically distinct solutions.

Remark 1.1. The condition $\left(f_{5}\right)$ was first introduced by Bartsch and Mederski [2] to study the time-harmonic Maxwell equations and then as used in [12] for a Schrödinger system where our first conclusion was covered. In fact, this condition can be regarded as a vector version of the following monotonicity condition :

$$
u \mapsto \frac{f(x, u)}{|u|} \text { is strictly increasing on }(-\infty, 0) \text { and on }(0, \infty),
$$

so that assumptions $\left(f_{1}\right)-\left(f_{5}\right)$ are more general than those in [21].

Remark 1.2. Here is an example which satisfies the assumptions $\left(f_{1}\right)-\left(f_{5}\right)$. For example, $F(x, u)=\Gamma(x)|M u|^{q}$ for $q \in\left(2,2^{*}\right)$, where $\Gamma \in L^{\infty}\left(\mathbb{R}^{N}\right)$ is 1-periodic in $x_{1}, \ldots, x_{N}$, positive, and bounded away from $0, M \in G L(2)$ is an invertible $2 \times 2$ matrix.

In the present paper, we are concerned with the existence of infinitely many solutions of (1.1). The main technical difficulty we need to overcome is that the energy functional is strongly indefinite. On the other hand, since $f$ is only continuous, $\mathcal{M}$ (see Section 2) may not be of class $C^{1}$ in our case, so we cannot use standard arguments on the Nehari manifold in the standard way. To overcome the nondifferentiability of the Nehari manifold, we shall use the reduction method developed by Szulkin and Weth [21,22].

2. Variational setting and preliminaries. First, $\left(f_{2}\right)-\left(f_{3}\right)$ imply that for every $\varepsilon>0$, there is $C_{\varepsilon}>0$ such that, for all $(x, u) \in \mathbb{R}^{N} \times \mathbb{R}^{2}$,

$$
|f(x, u)| \leq \varepsilon|u|+C_{\varepsilon}|u|^{p-1} \text { and }|F(x, u)| \leq \varepsilon|u|^{2}+C_{\varepsilon}|u|^{p} .
$$

Since $0 \notin \sigma\left(-\Delta+V_{i}\right)$, the spectral theory implies that there exist continuous projections $P_{i}^{+}$and $P_{i}^{-}$onto $E_{i}^{+}$and $E_{i}^{-}$, respectively, such that $H^{1}\left(\mathbb{R}^{N}\right)=$ $E_{i}^{+} \oplus E_{i}^{-}$for $i=1,2$ (see [17]). Denote $P_{i}^{ \pm} u=u_{i}^{ \pm}$for $u \in H^{1}\left(\mathbb{R}^{N}\right)$ in the sequel. Moreover, we introduce new inner products in $H^{1}\left(\mathbb{R}^{N}\right)$ by

$$
\langle u, v\rangle_{i}:=\int_{\mathbb{R}^{N}} \nabla u_{i}^{+} \nabla v_{i}^{+}+V_{i}(x) u_{i}^{+} v_{i}^{+} d x-\int_{\mathbb{R}^{N}} \nabla u_{i}^{-} \nabla v_{i}^{-}+V_{i}(x) u_{i}^{-} v_{i}^{-} d x
$$

and norms given by $\|u\|_{i}:=\left(\langle u, u\rangle_{i}\right)^{\frac{1}{2}}$ for $i=1,2$, which are equivalent to the $H^{1}$-norm. Let

$$
E^{+}:=E_{1}^{+} \times E_{2}^{+}, E^{-}:=E_{1}^{-} \times E_{2}^{-},
$$


and observe that any $u \in E:=H^{1}\left(\mathbb{R}^{N}\right) \times H^{1}\left(\mathbb{R}^{N}\right)$ admits a unique decomposition $u=u^{+}+u^{-}$, where $u^{+}=\left(u_{1}^{+}, u_{2}^{+}\right) \in E^{+}$and $u^{-}=\left(u_{1}^{-}, u_{2}^{-}\right) \in E^{-}$. We introduce a new norm in $E$ given by

$$
\|u\|^{2}=\sum_{i=1}^{2}\left(\left\|u_{i}^{+}\right\|_{i}^{2}+\left\|u_{i}^{-}\right\|_{i}^{2}\right)=\sum_{i=1}^{2}\left\|u_{i}\right\|_{i}^{2} .
$$

Thus, the energy functional $\Phi$ corresponding to (1.1) is given by

$$
\begin{aligned}
\Phi(u) & =\frac{1}{2} \sum_{i=1}^{2} \int_{\mathbb{R}^{N}}\left|\nabla u_{i}\right|^{2}+V_{i}(x)\left|u_{i}\right|^{2} d x-\int_{\mathbb{R}^{N}} F(x, u) d x \\
& =\frac{1}{2} \sum_{i=1}^{2}\left(\left\|u_{i}^{+}\right\|_{i}^{2}-\left\|u_{i}^{-}\right\|_{i}^{2}\right)-\int_{\mathbb{R}^{N}} F(x, u) d x \\
& =\frac{1}{2}\left\|u^{+}\right\|^{2}-\frac{1}{2}\left\|u^{-}\right\|^{2}-\int_{\mathbb{R}^{N}} F(x, u) d x,
\end{aligned}
$$

which is of $C^{1}$-class and its critical points correspond to solutions of (1.1).

In order to look for the ground state solutions of system (1.1), we consider the following set

$$
\mathcal{M}:=\left\{u \in E \backslash E^{-}: \Phi^{\prime}(u) u=0 \text { and } \Phi^{\prime}(u) v=0 \text { for all } v \in E^{-}\right\},
$$

which has been introduced by Pankov [13]. Clearly, $\mathcal{M}$ contains all nontrivial critical points of $\Phi$.

We consider the following minimizing problem:

$$
c_{0}:=\inf _{u \in \mathcal{M}} \Phi(u) .
$$

Since $c_{0}$ is the lowest level for $\Phi$ at which there are nontrivial solutions of (1.1), $u_{0}$ will be called a least energy solution or ground state solution.

Before proving our result, we need some preliminary lemmas.

Lemma 2.1. Let $u \in E, v \in E^{-}$, and $s \geq 0$ with $u \neq s u+v$, then

$$
\Phi(u)>\Phi(s u+v)-\Phi^{\prime}(u)\left(\frac{s^{2}-1}{2} u+s v\right) .
$$

Proof. Let $u, v$, and $s$ be as in the statement. Then we need to show that

$$
\Phi(s u+v)-\Phi^{\prime}(u)\left(\frac{s^{2}-1}{2} u+s v\right)-\Phi(u)=-\frac{1}{2}\|v\|^{2}+\int_{\mathbb{R}^{3}} \varphi(s, x) d x<0,
$$

where

$$
\varphi(s, x):=f(x, u)\left(\frac{s^{2}-1}{2} u+s v\right)+F(x, u)-F(x, s u+v) .
$$

We first claim that $\varphi(s, x) \leq 0$ for $s \geq 0$ and $x \in \mathbb{R}^{N}$. Without loss of generality, we assume that $u \neq 0$. Then by $\left(f_{4}\right)$, we have $\varphi(0, x) \leq 0$ and it follows from $\left(f_{2}\right)$ that

$$
\lim _{s \rightarrow \infty} \varphi(s, x)=-\infty .
$$


Let $s_{0} \geq 0$ be such that $\varphi\left(s_{0}, x\right)=\max _{s \geq 0} \varphi(s, x)$. We may assume that $s_{0}>0$ and thus $\partial_{s} \varphi\left(s_{0}, x\right)=0$. Therefore,

$$
f(x, u)\left(s_{0} u+v\right)=f\left(x, s_{0} u+v\right) u .
$$

If $f(x, u)\left(s_{0} u+v\right) \leq 0$, then by $\left(f_{4}\right)$,

$$
\begin{aligned}
\varphi\left(s_{0}, x\right) & =\frac{-s_{0}^{2}-1}{2} f(x, u) u+s_{0} f(x, u)\left(s_{0} u+v\right)+F(x, u)-F\left(x, s_{0} u+v\right) \\
& \leq-\frac{s_{0}^{2}}{2} f(x, u) u-F\left(x, s_{0} u+v\right) \\
& \leq 0 .
\end{aligned}
$$

If $f(x, u)\left(s_{0} u+v\right)>0$, then by $\left(f_{5}\right)$,

$$
\begin{aligned}
\varphi\left(s_{0}, x\right) & \leq f(x, u)\left(\frac{s_{0}^{2}-1}{2} u+s_{0} v\right)+\frac{(f(x, u) u)^{2}-\left(f(x, u)\left(s_{0} u+v\right)\right)^{2}}{2 f(x, u) u} \\
& =-\frac{(f(x, u) v)^{2}}{2 f(x, u) u} \\
& \leq 0 .
\end{aligned}
$$

Then we infer that $\varphi(s, x) \leq 0$ for any $s \geq 0$ and $x \in \mathbb{R}^{N}$. If $v \neq 0$, then $(2.2)$ holds.

Now we consider the case $v=0$. If there exists $s_{0}>0$ and $s_{0} \neq 1$ such that $\varphi\left(s_{0}, x\right)=\max _{s \geq 0} \varphi(s, x)$, then $\partial_{s}\left(s_{0}, x\right)=s_{0} f(x, u) u-f\left(x, s_{0} u\right) u=0$. Thus, it follows from $\left(f_{5}\right)$ that

$$
\begin{aligned}
\varphi\left(s_{0}, x\right) & =\frac{s_{0}^{2}-1}{2} f(x, u) u+F(x, u)-F\left(x, s_{0} u\right) \\
& <\frac{s_{0}^{2}-1}{2} f(x, u) u+\frac{(f(x, u) u)^{2}-\left(f(x, u)\left(s_{0} u\right)\right)^{2}}{2 f(x, u) u} \\
& =0,
\end{aligned}
$$

which implies that $\varphi(s, x)$ has only one maximum point at $s=1$. Therefore, $\varphi(s, x)<\varphi(1, x)=0$.

From Lemma 2.1, we have the following lemma.

Lemma 2.2. Let $u \in \mathcal{M}, v \in E^{-}$, and $s \geq 0$ with $u \neq s u+v$, then

$$
\Phi(u)>\Phi(s u+v) \text {. }
$$

We define for any $u \in E^{+} \backslash\{0\}$,

$$
\hat{E}(u):=E^{-} \oplus \mathbb{R}^{+} u .
$$

Thus, Lemma 2.2 implies that $u$ is the unique global maximum of $\left.\Phi\right|_{\hat{E}(u)}$. Applying Lemma 2.2, we can prove the following results, we omit the proof here.

Lemma 2.3. (i) There is a constant $\rho>0$ such that $\inf _{\mathcal{M}} \Phi \geq \inf _{S_{\rho}} \Phi>0$, where $S_{\rho}=\left\{u \in E^{+}:\|u\|=\rho\right\}$. 
(ii) $\left\|u^{+}\right\| \geq \max \left\{\sqrt{2 c_{0}},\left\|u^{-}\right\|\right\}>0$ for every $u \in \mathcal{M}$.

Lemma 2.4. If $\mathcal{W}$ is a compact subset of $E^{+} \backslash\{0\}$, then there exists $R>0$ such that $\Phi<0$ on $E(u) \backslash B_{R}(0)$ for each $u \in \mathcal{W}$.

Proof. Assume that this is not true. Then there exist sequences $\left\{u_{n}\right\} \subset \mathcal{W}$ and $w_{n} \in E\left(u_{n}\right)$ such that $\Phi\left(w_{n}\right) \geq 0$ for all $n \in \mathbb{N}$ and $\left\|w_{n}\right\| \rightarrow \infty$ as $n \rightarrow \infty$. By the compactness of $\mathcal{W}$, we can assume that $u_{n} \rightarrow u \in \mathcal{W},\|u\|=1$. Set $v_{n}=\frac{w_{n}}{\left\|w_{n}\right\|}=s_{n} u_{n}+v_{n}^{-}$, then

$$
0 \leq \frac{\Phi\left(w_{n}\right)}{\left\|w_{n}\right\|^{2}}=\frac{1}{2}\left(s_{n}^{2}-\left\|v_{n}^{-}\right\|^{2}\right)-\int_{\mathbb{R}^{N}} \frac{F\left(x, w_{n}\right)}{\left|w_{n}\right|^{2}}\left|v_{n}\right|^{2} d x,
$$

which implies that $\left\|v_{n}^{-}\right\|^{2} \leq s_{n}^{2}=1-\left\|v_{n}^{-}\right\|^{2}$. So $\frac{1}{\sqrt{2}} \leq s_{n} \leq 1$ and then, up to a subsequence, $s_{n} \rightarrow s>0, v_{n} \rightarrow v$, and $v_{n}(x) \rightarrow v(x)$ for a.e. $x \in \mathbb{R}^{N}$. Thus $v=s u+v^{-} \neq 0$. Set $\Omega:=\left\{x \in \mathbb{R}^{N}: v(x) \neq 0\right\}$. Then meas $(\Omega)>0$. Hence, for $x \in \Omega,\left|w_{n}(x)\right| \rightarrow+\infty$. Consequently, by Fatou's lemma, one has

$$
\int_{\mathbb{R}^{N}} \frac{F\left(x, w_{n}\right)}{\left|w_{n}\right|^{2}}\left|v_{n}\right|^{2} d x \geq \int_{\Omega} \frac{F\left(x, w_{n}\right)}{\left|w_{n}\right|^{2}}\left|v_{n}\right|^{2} d x \rightarrow \infty,
$$

which is a contradiction to (2.3). This completes the proof.

As a consequence of Lemmas 2.2-2.4, one has

Lemma 2.5. For any $u \in E \backslash E^{-}$, the set $\mathcal{M} \cap \hat{E}(u)$ consists of precisely one point $\hat{m}(u)$ which is the unique global maximum of $\left.\Phi\right|_{\hat{E}(u)}$.

Lemma 2.6. $\Phi$ is coercive on $\mathcal{M}$, i.e., $\Phi(u) \rightarrow \infty$ as $\|u\| \rightarrow \infty, u \in \mathcal{M}$.

Proof. If the conclusion is false, then there exist a sequence $\left\{u_{n}\right\} \subset \mathcal{M}$ such that $\left\|u_{n}\right\| \rightarrow \infty$ and $\Phi\left(u_{n}\right) \leq d$ for some $d \in\left[c_{0}, \infty\right)$. Let $v_{n}=\frac{u_{n}}{\left\|u_{n}\right\|}$. Then, up to a subsequence, $v_{n} \rightarrow v$ in $E$ and $v_{n}(x) \rightarrow v(x)$ for a.e. in $x \in \mathbb{R}^{N}$. By Lemma 2.3(ii), $\left\|v_{n}^{+}\right\|^{2} \geq \frac{1}{2}$. By Lions' concentration principle [11, Lemma 1.1], it is not difficult to check that $\left\{v_{n}^{+}\right\}$is nonvanishing, that is, there exist $r, \delta>0$ and a sequence $\left\{y_{n}\right\} \subset \mathbb{R}^{N}$ such that

$$
\int_{B_{r}\left(y_{n}\right)}\left|v_{n}^{+}\right|^{2} d x \geq \delta .
$$

By the assumptions of periodicity, we may assume that $\left\{y_{n}\right\}$ is bounded in $\mathbb{Z}^{N}$. Thus, up to a subsequence, one has $v_{n}^{+} \rightarrow v^{+}$in $L_{l o c}^{2}\left(\mathbb{R}^{N}\right)$ with $v^{+} \neq 0$. Set $\Omega=\left\{x \in \mathbb{R}^{N}: v(x) \neq 0\right\}$. Then meas $(\Omega) \neq 0$ and $\left|u_{n}(x)\right| \rightarrow \infty$ for each $x \in \Omega$. It follows from $\left(f_{3}\right)$ and Fatou's lemma that

$$
\int_{\mathbb{R}^{N}} \frac{F\left(x, u_{n}\right)}{\left\|u_{n}\right\|^{2}} d x \geq \int_{\Omega} \frac{F\left(x, u_{n}\right)}{\left|u_{n}\right|^{2}}\left|v_{n}\right|^{2} d x \rightarrow \infty,
$$

and therefore,

$$
0 \leq \frac{\Phi\left(u_{n}\right)}{\left\|u_{n}\right\|^{2}}=\frac{1}{2}\left(\left\|v_{n}^{+}\right\|^{2}-\left\|v_{n}^{-}\right\|^{2}\right)-\int_{\mathbb{R}^{N}} \frac{F\left(x, u_{n}\right)}{\left\|u_{n}\right\|^{2}} d x \rightarrow-\infty
$$


as $n \rightarrow \infty$, a contradiction.

Lemma 2.7 (see [21]). The map $\hat{m}: E^{+} \backslash\{0\} \rightarrow \mathcal{M}$ is continuous, and the restriction of the map $\hat{m}$ to $S^{+}$is a homeomorphism with inverse given by

$$
\check{m}: \mathcal{M} \rightarrow S^{+}, \check{m}(u)=\frac{u^{+}}{\left\|u^{+}\right\|},
$$

where $S^{+}:=\left\{u \in E^{+}:\|u\|=1\right\}$.

Define the mapping $\hat{\Psi}: E^{+} \backslash\{0\} \rightarrow \mathbb{R}$ and $\Psi: S^{+} \rightarrow \mathbb{R}$ by

$$
\hat{\Psi}(u)=\Phi(\hat{m}(u)) \text { and } \Psi=\left.\hat{\Psi}\right|_{S^{+}},
$$

which are continuous by Lemma 2.7.

By [21, Proposition 2.9 and Corollary 2.10], we have the following Lemma 2.8.

Lemma 2.8 (see [21]).

(i) $\hat{\Psi} \in C^{1}\left(E^{+} \backslash\{0\}, \mathbb{R}\right)$, and $\hat{\Psi}^{\prime}(w) z=\frac{\left\|\hat{m}(w)^{+}\right\|}{\|w\|} \Phi^{\prime}(\hat{m}(w)) z$ for $w, z \in E^{+}, w \neq$ 0 .

(ii) $\Psi \in \mathcal{C}^{1}\left(S^{+}, \mathbb{R}\right)$ and for each $w \in S^{+}$, one has

$\Psi^{\prime}(w) z=\left\|\hat{m}(w)^{+}\right\| \Phi^{\prime}(\hat{m}(w)) z$ for all $z \in T_{w}\left(S^{+}\right)=\left\{v \in E^{+}:\langle w, v\rangle=0\right\}$.

(iii) If $\left\{w_{n}\right\}$ is a $(P S)$-sequence for $\Psi$, then $\left\{\hat{m}\left(w_{n}\right)\right\}$ is a (PS)-sequence for $\Phi$.

(iv) $w \in S^{+}$is a critical point of $\Psi$ if and only if $\hat{m}(w) \in \mathcal{M}$ is a critical point of $\Phi$. Moreover, the corresponding values of $\Psi$ and $\Phi$ coincide and $\inf _{S^{+}} \Psi=\inf _{\mathcal{M}} \Phi$.

3. Proof of the main result. Now we are in a position to give the proof of existence in Theorem 1.1.

Proof of Theorem 1.1. (i) If $u_{0} \in \mathcal{M}$ satisfies $\Phi\left(u_{0}\right)=c_{0}$, then $\check{m}\left(u_{0}\right) \in S^{+}$ is a minimizer of $\Psi$ and therefore a critical point of $\Psi$, thus $u_{0}$ is a critical point of $\Phi$ by Lemma 2.8. It remains to show that there exists a minimizer $u \in \mathcal{M}$ of $\left.\Phi\right|_{\mathcal{M}}$. By Ekeland's variational principle [23], there exists a sequence $\left\{w_{n}\right\} \subset S^{+}$with $\Psi\left(w_{n}\right) \rightarrow c_{0}$ and $\Psi^{\prime}\left(w_{m}\right) \rightarrow 0$. Set $u_{n}=\hat{m}\left(w_{n}\right) \in \mathcal{M}$. Then $\Phi\left(u_{n}\right) \rightarrow c_{0}$ and $\Phi^{\prime}\left(u_{n}\right) \rightarrow 0$. By Lemma 2.6, $\left\{u_{n}\right\}$ is bounded in $E$. Therefore, up to a subsequence, $u_{n} \rightarrow u$ in $E$. By Lions' concentration principle [11, Lemma 1.1], it is not difficult to check that $\left\{u_{n}\right\}$ is nonvanishing, that is, there exist $r, \delta>0$ and a sequence $\left\{y_{n}\right\} \subset \mathbb{R}^{N}$ such that

$$
\int_{B_{r}\left(y_{n}\right)}\left|u_{n}\right|^{2} d x \geq \delta,
$$

here we may assume that $y_{n} \in \mathbb{Z}^{N}$ by taking a large $r$ if necessary. By the assumptions of periodicity, we may assume that $\left\{y_{n}\right\}$ is bounded in $\mathbb{Z}^{N}$. Thus, one has $u \neq 0, \Phi^{\prime}(u)=0$, and then $u \in \mathcal{M}$. Using a standard argument, one obtains that $\Phi(u)=c_{0}$. This completes the first part of Theorem 1.1. 
In the following, we focus on the proof of the multiplicity of Theorem 1.1. Set

$$
K:=\left\{u \in S^{+}: \Psi^{\prime}(u)=0\right\}, K_{d}:=\{u \in K: \Psi(u)=d\},
$$

and

$$
U_{\delta}\left(K_{d}\right):=\left\{u \in S^{+}: \operatorname{dist}\left(u, K_{d}\right)<\delta\right\} .
$$

Let $\Sigma:=\left\{A \subset S^{+}: A\right.$ is closed and $\left.A=-A\right\}$. For each $A \in \Sigma, \gamma(A)$ denotes the Krasnoselskii genus (see $[15,20]$ ) of $A$, which is defined as the least integer $k$ such that there exists an odd continuous mapping $\sigma: A \rightarrow \mathbb{R}^{k} \backslash\{0\}$. If there is no such mapping for any $k$, then $\gamma(A)=\infty$. Moreover, $\gamma(\emptyset)=0$. Set

$$
c_{k}:=\inf \left\{d \in \mathbb{R}: \gamma\left(\Psi^{d}\right) \geq k\right\}
$$

for all $k \in \mathbb{N}$. It is easy to prove that $c_{0} \leq c_{k}$ and $c_{k} \leq c_{k+1}$.

Lemma 3.1. $c_{k}$ is a critical value of $\Psi$.

Proof. If $c_{k}$ is not a critical value of $\Psi$, then for any $w \in S^{+}$, one has $\Psi(w) \neq c_{k}$ or $\Psi^{\prime}(w) \neq 0$. Hence, there exists $\delta>0$ such that

$$
N_{c_{k}, \delta}:=\left\{w \in S^{+}:\left|\Psi(w)-c_{k}\right|<\delta,\left\|\Psi^{\prime}(w)\right\|<\delta\right\}=\emptyset .
$$

Otherwise, there exists a sequence $\left\{w_{n}\right\} \subset S^{+}$such that $\Psi\left(w_{k}\right) \rightarrow c_{k}$ and $\left\|\Psi^{\prime}\left(w_{n}\right)\right\| \rightarrow 0$. Set $v_{n}=\hat{m}\left(w_{n}\right)$. Then, by Lemma 2.8(iii), $\left\{v_{n}\right\} \subset \mathcal{M}$ is a $(P S)_{c_{k}}$ sequence of $\Phi$. Lemma 2.6 implies that $\left\{v_{n}\right\}$ is bounded in $E$. Hence, up to a subsequence, one has $u_{n} \rightarrow u$ in $E, u_{n} \rightarrow u$ in $L_{l o c}^{t}\left(\mathbb{R}^{N}\right)$ for all $t \in\left[1,2^{*}\right)$, and $u_{n}(x) \rightarrow u(x)$ for a.e. $x \in \mathbb{R}^{N}$. As in the proof of Theorem 1.1, we can prove that $\Phi^{\prime}(v)=0$ and $v_{n} \rightarrow v$ in $E$, and hence $\Phi(v)=c_{k} \geq c_{0}>0$. Consequently, $v \in \mathcal{M}$, and hence Lemma 2.8(iii) implies $w:=m^{-1}(v) \in K_{c_{k}}$, a contradiction. This show $N_{c_{k}, \delta}=\emptyset$. Therefore, by [20, Remark $\left.\amalg 3.12\right]$, there exists $\varepsilon_{0}>0$ such that for any $0<\varepsilon<\bar{\varepsilon} \leq \varepsilon_{0}$, and there exists a continuous 1-parameter family of homeomorphisms $\eta(t, \cdot)$ of $S^{+}, 0 \leq t<\infty$, with the properties:

$\left(1^{\circ}\right) \eta(w, t)=w$ if $t=0$, or $\Psi^{\prime}(w)=0$, or $\left|\Psi(w)-c_{k}\right| \geq \bar{\varepsilon}$;

$\left(2^{\circ}\right) \Psi(\eta(w, t))$ is nonincreasing in $t$ for any $w \in S^{+}$;

$\left(3^{\circ}\right) \eta\left(\Psi^{c_{k}+\varepsilon}, 1\right) \subset \Psi^{c_{k}-\varepsilon}$

$\left(4^{\circ}\right) \eta(\cdot, s) \circ \eta(\cdot, t)=\eta(\cdot, s+t)$ for all $s, t \geq 0$;

$\left(5^{\circ}\right) \eta(w, t)$ is odd in $w$ for $t \geq 0$.

Moreover, by $N_{c_{k}, \delta}=\emptyset$, we know that there exists $0<\varepsilon_{1}<\varepsilon_{0}$ such that

$$
\Psi_{c_{k}-\varepsilon_{1}}^{c_{k}+\varepsilon_{1}} \bigcap K=\emptyset \text {. }
$$

For each $w \in \Psi^{c_{k}+\varepsilon_{1}}$, by the property $\left(3^{\circ}\right)$ of $\eta$, we know that $\Psi(\eta(w, 1)) \leq$ $c_{k}-\varepsilon_{1}$. Let $e=e(w)$ be the infimum of the time for which $\Psi(\eta(w, t)) \leq c_{k}-\varepsilon_{1}$. It is easy to see that $e: \Psi^{c_{k}+\varepsilon_{1}} \rightarrow[0, \infty)$ is a continuous mapping. Since $\Psi$ is even, so is $e$. Define a mapping $h: \Psi^{c_{k}+\varepsilon_{1}} \rightarrow \Psi^{c_{k}-\varepsilon_{1}}$ by $h(w):=\eta(w, e(w))$. Then $h$ is odd and continuous. It follows from the mapping property of the genus and the definition of $c_{k}$ that

$$
k \leq \gamma\left(\Psi^{c_{k}+\varepsilon_{1}}\right) \leq \gamma\left(\Psi^{c_{k}-\varepsilon_{1}}\right) \leq k-1,
$$


a contradiction.

Now, set

$$
\tilde{K}:=\bigcup_{k=1}^{\infty} K_{c_{k}} .
$$

Choose a subset $\mathcal{F}$ of $\tilde{K}$ such that $\mathcal{F}=-\mathcal{F}$ and each orbit has a unique representation. Arguing indirectly, from now on, we always assume that

$$
\mathcal{F} \text { is a finite set. }
$$

The following lemma has been proved in [21, Lemma 2.13].

Lemma 3.2. $\kappa:=\inf \{\|v-w\|: v, w \in \tilde{K}, v \neq w\}>0$.

The following key lemma gives the discreteness property of $(P S)$-sequences of $\Phi$.

Lemma 3.3 (Discreteness of (PS)-sequences). Let $d \geq c_{0}$. If $\left\{v_{n}^{1}\right\},\left\{v_{n}^{2}\right\} \subset \Phi^{d}$ are two $(P S)$-sequences for $\Phi$, then either $\lim _{n \rightarrow \infty}\left\|v_{n}^{1}-v_{n}^{2}\right\|=0$ or $\limsup _{n \rightarrow \infty} \| v_{n}^{1}-$ $v_{n}^{2} \| \geq \rho(d)>0$, where $\rho(d)$ depends only on $d$ but not on the particular choice of the $(P S)$-sequences.

Proof. We put $u_{n}^{1}:=\hat{m}\left(v_{n}^{1}\right), u_{n}^{2}:=\hat{m}\left(v_{n}^{2}\right)$. Then by Lemmas 2.8(iii) and 2.6, both sequences $\left\{u_{n}^{1}\right\},\left\{u_{n}^{2}\right\} \in \Phi^{d} \cap \mathcal{M}$ are bounded $(P S)$-sequence for $\Phi$. We distinguish two cases.

Case $1\left\{u_{n}^{1}-u_{n}^{2}\right\}$ is vanishing. Then $\left\|u_{n}^{1}-u_{n}^{2}\right\|_{p} \rightarrow 0$ as $n \rightarrow \infty$. Thus, $\left\|\left(u_{n}^{1}-u_{n}^{2}\right)^{+}\right\|_{p} \rightarrow 0$ since the orthogonal projection of $E$ on $E^{+}$is continuous in the $L^{p}$-norm. By the Hölder inequality and (2.1), one has

$$
\begin{aligned}
\left\|\left(u_{n}^{1}-u_{n}^{2}\right)^{+}\right\|^{2}= & \Phi^{\prime}\left(u_{n}^{1}\right)\left(u_{n}^{1}-u_{n}^{2}\right)^{+}-\Phi^{\prime}\left(u_{n}^{2}\right)\left(u_{n}^{1}-u_{n}^{2}\right)^{+} \\
& +\int_{\mathbb{R}^{3}}\left(f\left(x, u_{n}^{1}\right)-f\left(x, u_{n}^{2}\right)\right)\left(u_{n}^{1}-u_{n}^{2}\right)^{+} d x \\
\leq & \varepsilon\left\|\left(u_{n}^{1}-u_{n}^{2}\right)^{+}\right\| \\
& +\int_{\mathbb{R}^{3}}\left(\varepsilon\left(\left|u_{n}^{1}\right|+\left|u_{n}^{2}\right|\right)+C_{\varepsilon}\left(\left|u_{n}^{1}\right|^{p-1}+\left|u_{n}^{2}\right|^{p-1}\right)\right)\left|\left(u_{n}^{1}-u_{n}^{2}\right)^{+}\right| d x \\
\leq & (1+C) \varepsilon\left\|\left(u_{n}^{1}-u_{n}^{2}\right)^{+}\right\|+D_{\varepsilon}\left\|\left(u_{n}^{1}-u_{n}^{2}\right)^{+}\right\|_{p},
\end{aligned}
$$

which implies that $\left\|\left(u_{n}^{1}-u_{n}^{2}\right)^{+}\right\| \rightarrow 0$ as $n \rightarrow \infty$. Similarly, $\left\|\left(u_{n}^{1}-u_{n}^{2}\right)^{-}\right\| \rightarrow 0$ as $n \rightarrow \infty$, so $\left\|u_{n}^{1}-u_{n}^{2}\right\| \rightarrow 0$ as $n \rightarrow \infty$. As a consequence, $\left\|v_{n}^{1}-v_{n}^{2}\right\| \rightarrow 0$ as $n \rightarrow \infty$ because $\check{m}$ is Lipschitz continuous on $\mathcal{M}$; indeed for $u, v \in \mathcal{M}$, by Lemma 2.3,

$$
\begin{aligned}
\|\check{m}(u)-\check{m}(v)\| & =\left\|\frac{u^{+}}{\left\|u^{+}\right\|}-\frac{v^{+}}{\left\|v^{+}\right\|}\right\|=\left\|\frac{u^{+}-v^{+}}{\left\|u^{+}\right\|}-\frac{\left(\left\|u^{+}\right\|-\left\|v^{+}\right\|\right) v^{+}}{\left\|u^{+}\right\|\left\|v^{+}\right\|}\right\| \\
& \leq \frac{2}{\left\|u^{+}\right\|}\left\|(u-v)^{+}\right\| \leq \sqrt{\frac{2}{c_{0}}}\|u-v\| .
\end{aligned}
$$


Case $2\left\{u_{n}^{1}-u_{n}^{2}\right\}$ is nonvanishing. That is, there exist $r, \delta>0$ and a sequence $\left\{y_{n}\right\} \subset \mathbb{R}^{N}$ such that

$$
\int_{B_{r}\left(y_{n}\right)}\left|u_{n}^{1}-u_{n}^{2}\right|^{2} d x \geq \delta
$$

By the assumptions of periodicity, we may assume that $\left\{y_{n}\right\}$ is bounded in $\mathbb{R}^{N}$. Up to a subsequence, we may assume that $u_{n}^{1} \rightarrow u^{1}$ and $u_{n}^{2} \rightarrow u^{2}$ in $E$, where $u^{1} \neq u^{2}$ by $(3.1)$ and $\Phi^{\prime}\left(u^{1}\right)=\Phi^{\prime}\left(u^{2}\right)=0$. Now we suppose

$$
\left\|u_{n}^{1}\right\| \rightarrow \alpha^{1} \text { and }\left\|u_{n}^{2}\right\| \rightarrow \alpha^{2},
$$

then it follows from Lemma 2.3(ii) that $\sqrt{2 c_{0}} \leq \alpha_{i} \leq \nu(d):=\sup \{\|u\|: u \in$ $\left.\Phi^{d} \cap \mathcal{M}\right\}$ (note that $\nu(d)<\infty$ by Lemma 2.6) for $i=1,2$.

Suppose $u^{1}, u^{2} \neq 0$. Then $u^{1}, u^{2} \in \mathcal{M}$. We put $v^{1}:=\check{m}\left(u^{1}\right) \in K, v^{2}:=$ $\check{m}\left(u^{2}\right) \in K, v^{1} \neq v^{2}$. Hence

$$
\begin{aligned}
& \liminf _{n \rightarrow \infty}\left\|v_{n}^{1}-v_{n}^{2}\right\|=\liminf _{n \rightarrow \infty}\left\|\frac{\left(v_{n}^{1}\right)^{+}}{\left\|\left(v_{n}^{1}\right)^{+}\right\|}-\frac{\left(v_{n}^{2}\right)^{+}}{\left\|\left(v_{n}^{2}\right)^{+}\right\|}\right\| \\
& \geq\left\|\frac{\left(u^{1}\right)^{+}}{\alpha^{1}}-\frac{\left(u^{2}\right)^{+}}{\alpha^{2}}\right\| \geq\left\|\beta_{1} v_{1}-\beta_{2} v_{2}\right\|,
\end{aligned}
$$

where

$$
\beta_{1}:=\frac{\left\|\left(u^{1}\right)^{+}\right\|}{\alpha_{1}} \geq \frac{\sqrt{2 c_{*}}}{\nu(d)} \text { and } \beta_{2}:=\frac{\left\|\left(u^{2}\right)^{+}\right\|}{\alpha_{2}} \geq \frac{\sqrt{2 c_{*}}}{\nu(d)} .
$$

Since $\left\|v^{1}\right\|=\left\|v^{2}\right\|=1$, it is easy see from the above inequalities that

$$
\liminf _{n \rightarrow \infty}\left\|v_{n}^{1}-v_{n}^{2}\right\| \geq\left\|\beta_{1} v^{1}-\beta_{2} v^{2}\right\| \geq \min \left\{\beta^{1}, \beta^{2}\right\}\left\|v^{1}-v^{2}\right\| \geq \frac{\kappa \sqrt{2 c_{0}}}{\nu(d)}
$$

where $\kappa$ is given by Lemma 3.2. Hence (3.2) implies $\liminf _{n \rightarrow \infty}\left\|v_{n}^{1}-v_{n}^{2}\right\| \geq \rho(d)>$ 0 , where $\rho(d)$ depends only on $d$.

If $u^{2}=0$, then $u^{1} \neq 0$ and

$$
\liminf _{n \rightarrow \infty}\left\|v_{n}^{1}-v_{n}^{2}\right\|=\liminf _{n \rightarrow \infty}\left\|\frac{\left(v_{n}^{1}\right)^{+}}{\left\|\left(v_{n}^{1}\right)^{+}\right\|}-\frac{\left(v_{n}^{2}\right)^{+}}{\left\|\left(v_{n}^{2}\right)^{+}\right\|}\right\| \geq \frac{\left\|\left(u^{1}\right)^{+}\right\|}{\alpha^{1}} \geq \frac{\sqrt{2 c_{0}}}{\nu(d)} .
$$

The case $u^{1}=0$ can be treated similarly. The proof is completed.

It is known that $\Psi$ admits a pseudo-gradient vector field $H: S^{+} \backslash K \rightarrow T S^{+}$. Let $\eta_{1}: \mathcal{G} \rightarrow S^{+} \backslash K$ be the corresponding flow defined by

$$
\left\{\begin{array}{l}
\frac{d}{d t} \eta_{1}(t, w)=-H\left(\eta_{1}(t, w)\right) \\
\eta_{1}(0, w)=w
\end{array}\right.
$$

where

$$
\mathcal{G}:=\left\{(t, w): w \in S^{+} \backslash K, T^{-}(w)<t<T^{+}(w)\right\} \subset \mathbb{R} \times\left(S^{+} \backslash K\right)
$$

and $\left(T^{-}(w), T^{+}(w)\right)$ are the maximal existence times of the trajectory $t \rightarrow$ $\eta_{1}(t, w)$ in negative and positive direction. Here $\eta_{1}(t, w)$ is odd in $w$ and $\Psi\left(\eta_{1}(t, w)\right)$ is strictly decreasing in $t$. 
We then have the following important deformation type results which play a crucial role in our proof.

Lemma 3.4. Let $d \geq c_{0}$. Then for every $\delta>0$, there exists $\varepsilon=\varepsilon(\delta)>0$ such that

(i) $\Psi_{d-\varepsilon}^{d+\varepsilon} \cap \tilde{K}=K_{d}$;

(ii) $\lim _{t \rightarrow T^{+}(w)} \Psi\left(\eta_{1}(t, w)\right)<d-\varepsilon$ for $w \in \Psi^{d+\varepsilon} \backslash U_{\delta}\left(K_{d}\right)$.

Proof of Theorem 1.1 (completed). (ii) If $\mathcal{F}$ is an infinite set, then $\Psi$ admits infinitely many pairs $\pm v$ of geometrically distinct critical points. So, Theorem 1.1(ii) follows from Lemmas 2.8 and 3.1.

By Lemma $3.2, K_{c_{k}}$ is either empty or a discrete set, hence $\gamma\left(K_{c_{k}}\right)=0$ or 1. By the continuity property of the genus, there exists $\delta>0$ such that $\gamma(\bar{U})=$ $\gamma\left(K_{c_{k}}\right)$, where $U:=U_{\delta}\left(K_{c_{k}}\right)$ and $\delta<\frac{\kappa}{2}$. For such $\delta$, choose $\varepsilon>0$ so that the conclusions of Lemma 3.4 hold with $d=c_{k}$. Then for each $w \in \Psi^{c_{k}+\varepsilon} \backslash U$, there exists $t \in\left[0, T^{+}(w)\right)$ such that $\Psi\left(\eta_{1}(t, w)\right)<c_{k}-\varepsilon$. Let $e=e(w)$ be the infimum of the time for which $\Psi\left(\eta_{1}(w, t)\right) \leq c_{k}-\varepsilon$. Since $c_{k}-\varepsilon$ is not a critical value of $\Psi$, it is easy to see by the implicit function theorem that $e$ is a continuous mapping and since $\Psi$ is even, $e(-w)=e(w)$. Define a mapping $h: \Psi^{c_{k}+\varepsilon} \backslash U \rightarrow \Psi^{c_{k}-\varepsilon}$ by setting $h(w):=\eta_{1}(e(w), w)$. Then $h$ is odd and continuous, so it follows from the properties of the genus and the definition of $c_{k}$ that

$$
\gamma\left(\Psi^{c_{k}+\varepsilon}\right) \leq \gamma(\bar{U})+\gamma\left(\Psi^{c_{k}-\varepsilon}\right) \leq \gamma(\bar{U})+k-1=\gamma\left(K_{c_{k}}\right)+k-1 .
$$

If $\gamma\left(K_{c_{k}}\right)=0$, then $\gamma\left(\Psi^{c_{k}+\varepsilon}\right) \leq k-1$, in contrast to the definition of $c_{k}$. Therefore, $\gamma\left(K_{c_{k}}\right)=1$ and $K_{c_{k}} \neq \emptyset$.

If $c_{k}=c_{k+1}$, then by the definition of $c_{k+1}$, there exists $r<c_{k+1}+\varepsilon$ such that $\gamma\left(\Psi^{r}\right) \geq k+1$. Therefore, $\gamma\left(\Psi^{c_{k+1}+\varepsilon}\right) \geq \gamma\left(\Psi^{r}\right) \geq k+1$, and hence, by (3.3), one has

$$
\gamma\left(K_{c_{k}}\right) \geq \gamma\left(\Psi^{c_{k}+\varepsilon}\right)-k+1 \geq 2 .
$$

But, by Lemma 3.2, $\gamma\left(K_{c_{k}}\right)=1$, a contradiction. Therefore, $c_{k}<c_{k+1}$. This contradicts the fact that $\mathcal{F}$ is a finite set. Therefore, $\Psi$ admits infinitely many pairs $\pm v$ of geometrically distinct critical points. Consequently, Theorem 1.1(ii) follows from Lemmas 2.8 and 3.1.

Acknowledgements. We would like to thank the anonymous referee for his/her careful readings of our manuscript and the useful comments on condition $\left(f_{5}\right)$. The first author also thanks the support of RTG 2419 by the German Science Foundation (DFG).

Funding Open Access funding provided by Projekt DEAL.

Open Access. This article is licensed under a Creative Commons Attribution 4.0 International License, which permits use, sharing, adaptation, distribution and reproduction in any medium or format, as long as you give appropriate credit to the original author(s) and the source, provide a link to the Creative Commons licence, and indicate if changes were made. The images or other third party material in 
this article are included in the article's Creative Commons licence, unless indicated otherwise in a credit line to the material. If material is not included in the article's Creative Commons licence and your intended use is not permitted by statutory regulation or exceeds the permitted use, you will need to obtain permission directly from the copyright holder. To view a copy of this licence, visit http://creativecommons. org/licenses/by/4.0/.

Publisher's Note Springer Nature remains neutral with regard to jurisdictional claims in published maps and institutional affiliations.

\section{References}

[1] Ambrosetti, A., Colorado, E.: Standing waves of some coupled nonlinear Schrödinger equations. J. Lond. Math. Soc. (2) 75(1), 67-82 (2007)

[2] Bartsch, T., Mederski, J.: Ground and bound state solutions of semilinear timeharmonic Maxwell equations in a bounded domain. Arch. Ration. Mech. Anal. 215(1), 283-306 (2015)

[3] Benci, V., Rabinowitz, P.H.: Critical point theorems for indefinite functionals. Invent. Math. 52(3), 241-273 (1979)

[4] Bonheure, D., Moreira dos Santos, E., Ramos, M.: Ground state and non-ground state solutions of some strongly coupled elliptic systems. Trans. Amer. Math. Soc. 364(1), 447-491 (2012)

[5] Clément, P.H., de Figueiredo, D.G., Mitidieri, E.: Positive solutions of semilinear elliptic systems. Comm. Partial Differ. Equ. 17(5-6), 923-940 (1992)

[6] de Figueiredo, D.G.: Semilinear elliptic systems: existence, multiplicity, symmetry of solutions. In: Handbook of Differential Equations: Stationary Partial Differential Equations, vol. V, Handb. Differ. Equ., pp. 1-48. Elsevier/NorthHolland, Amsterdam (2008)

[7] de Figueiredo, D.G., Felmer, P.L.: On superquadratic elliptic systems. Trans. Amer. Math. Soc. 343(1), 99-116 (1994)

[8] Ding, Y., Lee, C., Zhao, F.: Semiclassical limits of ground state solutions to Schrödinger systems. Calc. Var. Partial Differ. Equ. 51(3-4), 725-760 (2014)

[9] Ding, Y., Lin, F.: Semiclassical states of Hamiltonian system of Schrödinger equations with subcritical and critical nonlinearities. J. Partial Differ. Equ. 19(3), 232-255 (2006)

[10] Lin, T., Wei, J.: Spikes in two-component systems of nonlinear Schrödinger equations with trapping potentials. J. Differ. Equ. 229(2), 538-569 (2006)

[11] Lions, P.L.: The concentration-compactness principle in the calculus of variations. The locally compact case. II. Ann. Inst. H. Poincaré Anal. Non Linéaire 1(4), 223-283 (1984)

[12] Mederski, J.: Ground states of a system of nonlinear Schrödinger equations with periodic potentials. Comm. Partial Differ. Equ. 41(9), 1426-1440 (2016)

[13] Pankov, A.: Periodic nonlinear Schrödinger equation with application to photonic crystals. Milan J. Math. 73, 259-287 (2005) 
[14] Peng, S., Wang, Z.: Segregated and synchronized vector solutions for nonlinear Schrödinger systems. Arch. Ration. Mech. Anal. 208(1), 305-339 (2013)

[15] Rabinowitz, P.H.: Minimax methods in critical point theory with applications to differential equations. CBMS Regional Conference Series in Mathematics, vol. 65. Published for the Conference Board of the Mathematical Sciences, Washington, DC; by the American Mathematical Society, Providence, RI (1986)

[16] Ramos, M., Tavares, H.: Solutions with multiple spike patterns for an elliptic system. Calc. Var. Partial Differ. Equ. 31(1), 1-25 (2008)

[17] Reed, M., Simon, B.: Methods of Modern Mathematical Physics. IV. Analysis of Operators. Elsevier, New York (1978)

[18] Reed, M., Simon, B.: Methods of Modern Mathematical Physics. I. Academic Press Inc., New York (1980)

[19] Ruf, B.: Superlinear elliptic equations and systems. In: Handbook of Differential Equations: Stationary Partial Differential Equations, vol. V. Handb. Differ. Equ., pp. 211-276. Elsevier, Amsterdam (2008)

[20] Struwe, M.: Variational Methods. Applications to Nonlinear Partial Differential Equations and Hamiltonian Systems vol. 34, 3rd edn. Springer, Berlin (2000)

[21] Szulkin, A., Weth, T.: Ground state solutions for some indefinite variational problems. J. Funct. Anal. 257(12), 3802-3822 (2009)

[22] Szulkin, A., Weth, T.: The method of Nehari manifold. In: Handbook of Nonconvex Analysis and Applications, pp. 597-632. Int. Press, Somerville (2010)

[23] Willem, M.: Minimax Theorems, vol. 24. Birkhäuser Boston Inc., Boston (1996)

[24] Zhao, F., Ding, Y.: On Hamiltonian elliptic systems with periodic or non-periodic potentials. J. Differ. Equ. 249(12), 2964-2985 (2010)

[25] Zhao, L., Zhao, F., Shi, J.: Higher dimensional solitary waves generated by second-harmonic generation in quadratic media. Calc. Var. Partial Differ. Equ. 54(3), 2657-2691 (2015)

ZHIPENG YANG

Mathematical Institute

Georg-August-University of Göttingen

37073 Göttingen

Germany

e-mail: zhipeng.yang@mathematik.uni-goettingen.de

YUANYANG YU

Institute of Mathematics, Academy of Mathematics and Systems Science

Chinese Academy of Sciences

Beijing 100190

People's Republic of China

e-mail: yuyuanyang18@mails.ucas.ac.cn

and 
University of Chinese Academy of Sciences

Beijing 100049

People's Republic of China

Received: 1 February 2020 